\title{
Physics Students' Mental Model and Prediction in Heat Convection Concept
}

\author{
I. M. Sari, D. Saepuzzaman \\ Physics Education Department \\ Universitas Pendidikan Indonesia \\ Bandung, Indonesia \\ ikams@upi.edu
}

\begin{abstract}
Investigating students' mental model is something important in physics education. Mental model is students' internal representative of some phenomena that they find in daily life. A Student who has a scientifically mental model of a concept, he/she will be able to scientifically explain and predict the phenomena. Helping students construct scientifically mental model is one of obligation of college that produce physics teacher. The teacher who have the scientifically compatible mental model will create the physics learning meaningfully, thus the quality of physics learning will be improved. This study was first to excavate 23 physics students mental model of heat convection. Prediction of phenomena that relates to heat convection is probed to support that students run their mental model in predicting phenomena. A series of semi structured interviews was conducted to excavate the students' mental models and predictions f heat convection. The students who involved in this study come from different level from sophomore to master degree. The framework of assessing mental model and instrument adopted and adapted from Chiou [1]. We also compared the students' mental model and the relationship between mental model and prediction. The result shows that only 2 participants who have a scientifically mental model (model 7). Moreover, the relationships between mental models and predictions remains complex, this finding is appropriate with Chiou's finding. In predicting phenomena, students do not run their mental model.
\end{abstract}

Keywords-mental model, prediction

\section{INTRODUCTION}

One of challenge in physics learning is to help students construct a scientifically compatible understanding of physics phenomena based on their idea and belief [1]. Understanding that exist in someone thinking will help in describing the phenomena and process the understanding to analyze the new phenomena in the form of internal representation. Someone's internal representation based on his/her understanding of a concept is called Mental Model. The central goal in physics education is to help students construct a compatible scientifically mental model [2]

Excavating students' mental model in certain topic provides a constructive feedback to stakeholders of physics education particularly to probe the reasons behind misconception. This feedback is used to improve the quality of physics learning.
The concept of heat and heat convection is an essential concept and all physics students should master this concept as a basic concept.

Research in probing mental model of physics concept have been conducted by several researchers e.g.: Chiou [2] expressed the complex relationship between mental model and prediction. He suggested further research to investigate the relation between mental model and explanation. Chiou [2] results seven mental models in heat convection, that were diffusion-based convection model (model 1); gradualexpanding convection (model 2); Evenly distributed convection (model 3); warmness-topped convection (I) (model 4); Warmness-topped convection (II) (model 5); Rimcirculated convection (model 6); Fluent-cycled convection (model 7). Model 1 is a nave model and model 7 is a scientifically compatible model, but the order of these seven mental models does not imply a developing trend of scientific sophistication [2] Chiou categorized the students' mental model follow the framework shown in Fig. 1.

Another research in mental model was a research that conducted by Fazio, Ballaglia and Di Paola [3]. They investigated the relationship between the qualities of mental model with making of explanation. He suggested further qualitative and quantitative research in designing learning environment that focus to understanding of aspects in daily life at causation level and mechanism.

\section{RESEARCH PURPOSE}

The aim of this study was to identify college students' mental model in heat convection concept and investigated the relationship between mental model and prediction. The main goal of excavating mental model was to improve the quality of learning based on the depiction of students' conception and internal representation.

\section{RESEARCH METHODS}

Identified the college students mental model and investigated the relationship between mental model and prediction, this study used the qualitative research method. Moreover, the students' reasons behind their prediction were also explored to describe the relations in greater depth. A series of semi structured interviews were held to excavate students' mental model and the reasons behind the prediction. 
The students then were given follow up questions to strengthening the participants' prediction and the reasons why the participants make the prediction. At the end of the interviews, the participants were given an opportunity to reflect and/or to change the answers. Along the interviews, the participants' responses were recorded and transcribed to compare the participants' responses.

There were three interview protocols, first interview protocol contained basic concept of heat, heat convection. The second interview protocol contained four interview-aboutevent questions. One of interview-about-event question is shown in Fig. 2. The students were asked to predict the tubes that boil first.

The data of mental model was analyzed by constant comparison method. After that, the participants" response analyzed and compared to Chiou's framework. Three rounds of data analysis were conducted to construct each participant's mental model of heat convection [2] The first round focused to the participants drawing of phenomena; the second round focused to spatial arrangement and the last round was examining the reliability of patterns the students responses.

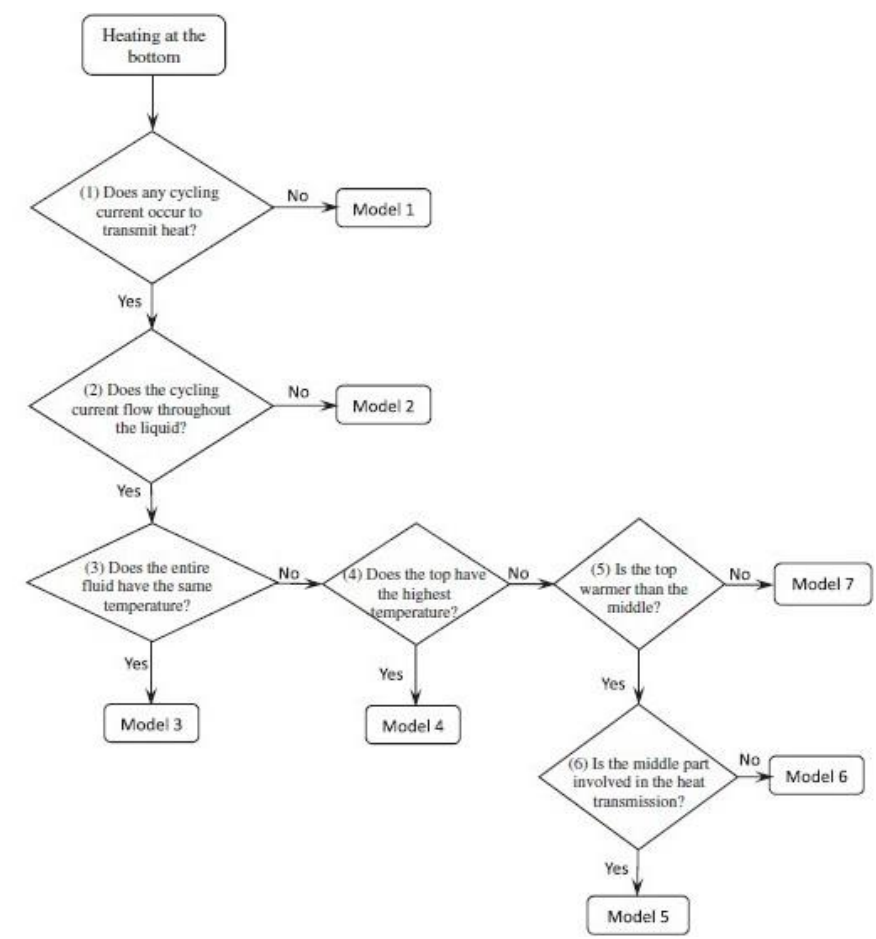

Fig. 1 Chiou's framework to differentiate students' mental model

A lecturer who has expertise in thermodynamics was asked to recode the participants' mental model and prediction. The intercoder reliability was calculated by Kappa coefficient which was $0.89(\mathrm{p}<0.05)$ and this was showing an excellent agreement between coders.

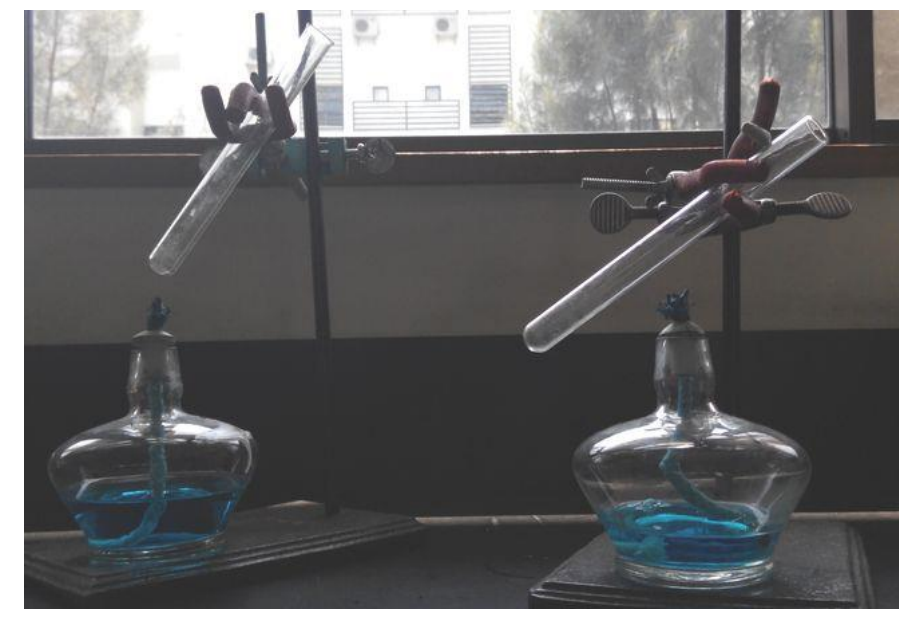

Fig. 2 Phenomena of heat convection

\section{RESULTS AND DISCUSSION}

\section{A. Authors and Affiliations}

Based on the students' response of the four interviewabout-event questions that recorded and referred to Chiou's framework to discriminate among pattern of students' response, the mental models of the participants showed in figure 3.

Model 1 was identified as Diffusion-based convection, which mechanism of heat transmission underlying in diffusion. Students who categorized as having this model, illustrated the heat as a substance, spreads from the heat source and gradually moves toward the cooler part; no current flows in this heat transmission [2]. Ten out of 23 students were in model 1, 5 students come from third semester (sophomore) who have not had assigned thermodynamics course. 5 students were from fifth and ninth semester and they had completed the course that related to thermal physics.

Surprisingly, 3 participants from master degree and 2-fresh graduate- participants were in model 3: Evenly distributed convection. This model has a central feature: equal temperature throughout a liquid at any moment of heat transmission. They thought that heat convection will reach the equilibrium of temperature in all parts of water particle. They did not consider about the interaction between the water particles in surface and the air particles.

Model 7, Fluent-cycled convection model is the closest to a scientifically compatible model. In this model the students able to explain the mechanism of heat convection microscopically. When the bottom part of fluid is heated, it becomes the warmest and least dense, and then starts to rise. $\mathrm{O}$ its way up, the rising current keeps releasing heat to its adjacent regions and thus gradually becomes cooler [2]. Based on Fig. 3, Only two students come from seventh semester (senior student college) and one fresh graduate participant who included to Model 7. 


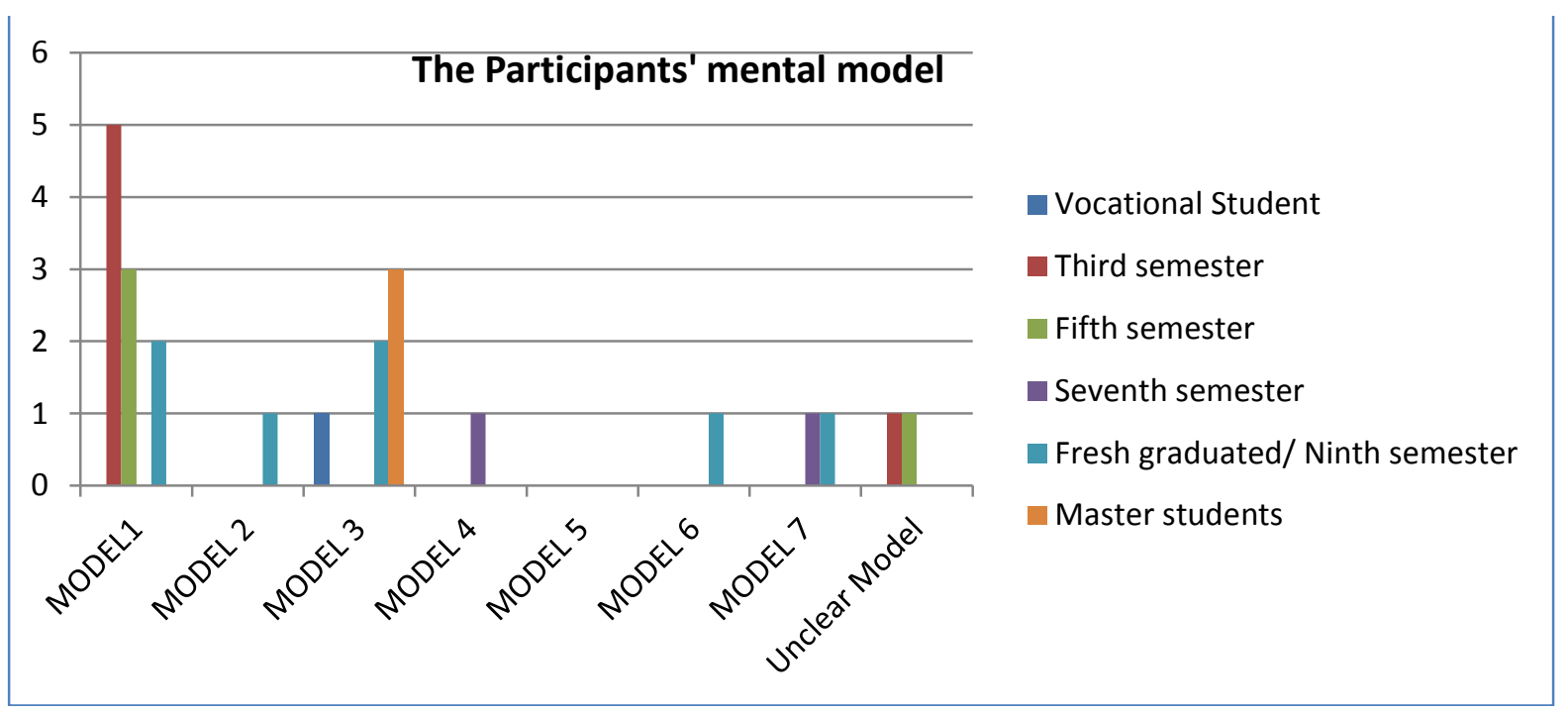

Fig. 3 The participants' mental model

There were not students who categorized as model 5 (Warmness-topped convection). All participants believe that the top of the liquid is not the hottest part of the liquid.

The figure 3 above shows us that there were not relationships between the seniority or level participants. We cannot differentiate whether the participants who had completed the thermodynamic course have an appropriate scientifically mental model or even the participants who had not completed the thermodynamic course have a nave model. This fact gives us a feedback to improve the quality of physics learning especially in heat transfer concept.

\section{B. Students' prediction}

The students were given the questions to predict which the tube will boil first if it is heated in different position. Tube 1 was heated in bottom of the tube; tube 2 was heated in center of the tube (see Fig. 2). The students' response showed in Table 1.

TABLE I. STUDENTS' PREDICTION

\begin{tabular}{lccc}
\hline \multicolumn{1}{c}{ Level } & Tube 1 & Tube 2 & $\begin{array}{l}\text { Both tubes } \\
\text { boil in the } \\
\text { same time }\end{array}$ \\
\hline Vocational student & 1 & 0 & 0 \\
Third semester students & 4 & 2 & 0 \\
Fifth semester students & 1 & 2 & 1 \\
Seventh semester students & 2 & 0 & 0 \\
Ninth semester students & 4 & 3 & 0 \\
Master Students & 0 & 1 & 2 \\
Total & 12 & 8 & 3 \\
\hline
\end{tabular}

Based on the results in table 1 , more than $50 \%$ of participants predicted tube 1 that will boil first. Several follow questions have been asked to participants to probe the reasons behind their choice. Most of them answered that the real mechanism of heat convection is heated at the bottom of the tube. Participant code A02 who chose Tube 1 gave a reason as follow:

I: "can you explain why you prefer Tube 1 than Tube 2 will boil first?"

A02: “I think...emm ... because in condition1, the lower part of water will hotter, then the heat will spread out to the upper part, so that tube 1 will be faster to boil, in contrast, Tube 2 the water that first hot is the water in the center (She pointed the part above the heat source), the hot water should spread first to the bottom of the tube and then to the surface. So tube 2 take a longer time to boil"

The participants who chose tube 2 that boil first, basically did not have the scientific reasons. Another word, they did not run their mental model. Table 2 shows the reasons from participants who chose Tube 2 .

TABLE II. PARTICIPANTS' REASON TO CHOSE TUBE 2

\begin{tabular}{cl}
$\begin{array}{c}\text { Students } \\
\text { code }\end{array}$ & \multicolumn{1}{c}{ Reason } \\
A05 & $\begin{array}{l}\text { The pressure experienced by fluid is less } \\
\text { Because in the tube 2, the movement of water particles } \\
\text { will move up and down, so that the spread process will } \\
\text { be faster in tube } 2 \\
\text { The different position of heat source, so that if it } \\
\text { heated, it will experience the different movement. So, } \\
\text { the movement is divided to up and down. }\end{array}$ \\
A11 & $\begin{array}{l}\text { Because of the heat source in tube } 2 \text { closer to edge, so } \\
\text { that the current is move upward and it will cause to } \\
\text { boil first }\end{array}$ \\
A07 & $\begin{array}{l}\text { Because heat is more spread to all the part of the tube } \\
\text { Boil is a condition that appear bubbles to the surface. } \\
\text { In tube 1, to reach the surface, its path is longer than }\end{array}$ \\
A15 &
\end{tabular}




\begin{tabular}{cl}
$\begin{array}{c}\text { Students' } \\
\text { code }\end{array}$ & \multicolumn{1}{c}{ Reason } \\
\hline & tube 2. So, tube 2 will boil firs \\
A17 & $\begin{array}{l}\text { There are two convection current, but at a time later, } \\
\text { all part of fluid will have a same temperature } \\
\text { Because the heat source is in the center of the tube, } \\
\text { then the heat will spread to bottom edge and upper } \\
\text { edge, its differ with tube 1 which the heat spread from } \\
\text { bottom to up, and it takes longer path. }\end{array}$ \\
\hline
\end{tabular}

\section{The relationship of mental model and predictions}

Ideally, someone who has a scientifically mental model and runs the mental model, he/she will be able to explain the phenomena and predict the phenomena scientifically. Chiou [2] expressed that running a mental model can be "read out" as the prediction for the final states of a given system. He also stated that mental models are capable of fulfilling a prediction.

This study found that there was a complex relationship between mental model and prediction (see Fig.4). One of participant who categorized as model 7 chooses Tube 1; while $50 \%$ of participants in model 1 also choose Tube 1 . All participants who categorized to Model 2 predicted Tube 1 that will boil first. In contrast, All participant who categorized as Model 6 prefer Tube 2 as the the tube that boil first. All participants, who thought that both tubes will boil in the same time, come from participants who are categorized as Model 3. Thus conditions describe a complex relationship between the participants' mental model and predictions. Most of participants did not run their mental model in predicting the phenomena. More than $50 \%$ of participants prefer to tube 1 that will boil first, their reasons are not based on the appropriate understanding, but it tend to common sense. In daily life, they often heating water or some food etc. with heat source is put in the bottom of the kettle.

The complexity of relationship between mental model and prediction is in line with the results of Chiou [2]. He addressed the complexity by two different levels of analysis that are the detailed level and a grand-scale perspective. At the detailed level, he found that the participants did not run the mental model in predicting the final states oh heat convection. At the grand-scale perspective, the participants' mental models of heat transmission did affect how they made the resultant prediction.

In contrast with this finding, no one participant was aware the entire process of heat transmission. They just thought that in liquid all heat be transmitted through convection. So, in describing the boiling mechanism in tube 2 , there is no one participant who answered that lower half tube 2 heat was transmitted through conduction and upper half through convection, so that tube 2 will boil first.

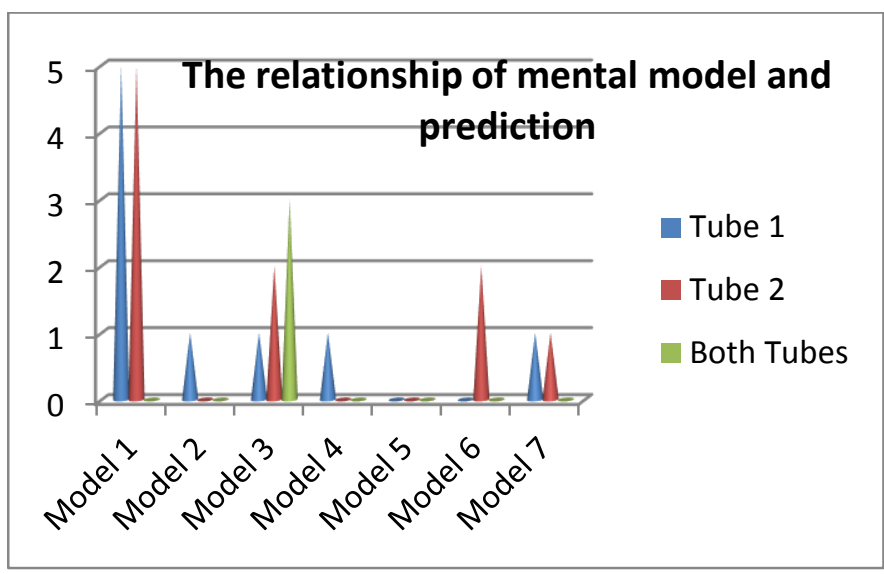

Fig. 4 The relationship of mental model and prediction

\section{CONCLUSION}

Based on this finding, we can conclude that the participants' mental model were 6 models from seven models found by Chiou [2]. There was not find participants who categorized as Model 5. The participants' prediction was not based on their running mental model. They tend to use common sense that the phenomena they found in the daily life was more likely the tube 1 , so most of the participants prefer tube 1 as the tube which boil first. This finding led us to improve the quality of learning especially in delivering heat concept as a dynamic and essential concept.

\section{ACKNOWLEDGMENT}

This study was supported by the Universitas Pendidikan Indonesia, under scheme: Hibah penelitian dan penguatan kompetensi , contracts No. 3858/UN40/LT/2015.

\section{REFERENCES}

[1] G.L. Chiou and O.R. Anderson, "A study of undergraduate physics students' understanding of heat conduction based on mental model theory and an model theory and an ontology-process analysis," Science Education Journal Volume 94 pp 825-854, 2010

[2] G.L. Chiou, "Reappraising the relationships between physics student's mental models and predictions: an example of heat convection,' Physical Review Special Topiks-Physics Education Research Volume 9, 2013.

[3] C. Fazio, O.R. Battaglia, and B.D. Paola, "Investigating the quality of mental models deployed by undergraduate engineering students in creating explanations: the case of thermally activated phenomena," Physical Review Special Topiks-Physics Education Research Volume 9 2013 